\title{
Intricate insights
}

DOI:

10.1038/nrc2039

\section{URLs}

Pancreatic cancer

http://www.cancer.gov/ cancertopics/types/pancreatic

\section{TGF $\beta$}

http://www.ncbi.nlm.nih.gov/ entrez/query.fcgi?db=gene\&c $\mathrm{md}=$ Retrieve\&dopt=full_ report\&list_uids $=7040$

\section{TGFBR2}

http://www.ncbi.nlm.nih.gov/ entrez/query.fcgi?db=gene\&c $\mathrm{md}=$ Retrieve\&dopt=full reportElist_uids=7048

\section{CDKN2A}

http://www.ncbi.nlm.nih.gov/ entrez/query.fcgi?db=gene\&c $\mathrm{md}=$ Retrieve\&dopt=full_ report\&list_uids=1029

SMAD4

http://www.ncbi.nlm.nih.gov/ entrez/query.fcgi?db=gene\&c $\mathrm{md}=$ Retrieve\&dopt=full_ reportElist_uids $=4089$

KRAS

http://www.ncbi.nlm.nih.gov/ entrez/query.fcgi?db=gene\&c $\mathrm{md}=$ Retrieve\&dopt=Graphics $\delta$ list_uids $=3845$
The effect of transforming growth factor- $\beta$ (TGF $\beta$ ) on tumour development and progression is complex. TGF $\beta$ can suppress the growth of early, precancerous lesions, but can also promote the growth of established carcinomas. The disruption of this pathway is evident in human pancreatic cancer, so two groups have generated mouse models of pancreatic carcinogenesis that involve the disruption of the TGF $\beta$ signalling pathway.

Mutation resulting in the activation of KRAS is thought to be an early event in the development of human pancreatic ductal adenocarcinomas (PDACs). As the tumours progress, loss of the tumour-suppressor gene CDKN2A (which encodes both the INK4a and ARF tumour suppressors) occurs, and this is accompanied by the loss of functional SMAD4 (an essential component of the TGF $\beta$ signalling pathway) in about $50 \%$ of cases. Nabeel Bardeesy, Kuang-hung Cheng, Ron de Pinho and colleagues used Smad4 conditional knockout mice to ablate SMAD4 expression specifically in pancreatic epithelial cells in mice that expressed activated KRAS.

In mice, the expression of a knock-in allele of activated Kras $\left(\right.$ Kras $\left.^{\mathrm{G} 12 \mathrm{D}}\right)$ induces the development of pancreatic intraepithelial neoplasias (PanINs; precursors of PDAC). The authors found that the loss of SMAD4 accelerated PDAC development in these mice. However, the loss of SMAD4 was more frequently associated with the development of intraductal papillary mucinous neoplasms (IPMNs), another potential precursor of PDAC. The combination of activated $\mathrm{Kras}^{\mathrm{G} 12 \mathrm{D}}$ with the loss of one allele of $C d k n 2 a$ and the loss of both alleles of Smad4 induced the rapid development of IPMNs that progressed to advanced PDACs. Therefore, the loss of Smad4 changes the histology of the precursor lesions, and the loss of $C d k n 2 a$ is required for full malignant progression to PDAC. The loss of Smad4 also altered the degree of differentiation seen in the tumours. Those without SMAD4 expression retained a differentiated phenotype, whereas tumours with intact SMAD4 expression were frequently associated with an epithelial-mesenchymal transition (EMT) phenotype. These mouse models indicate that SMAD4 functions to suppress initial tumour formation in response to activated KRAS, and in tumours in which SMAD4 remains expressed TGF $\beta$ can induce EMT and TGF $\beta$-dependent growth.

The loss or mutation of one of the TGF $\beta$ type II receptors, TGFBR2, is seen in a small subset of human PDACs. Harold Moses and colleagues have generated a mouse model in which Tgfbr 2 is specifically knocked out in pancreatic epithelial cells. Unlike the loss of Smad4, the combination of Tgfbr2 loss and the activation of $K r a s^{\mathrm{G} 12 \mathrm{D}}$ is sufficient to induce the formation of advanced PDACs through PanIN lesions in $100 \%$ of animals, and these tumours closely resemble the progression of human PDAC. SMAD4 was expressed normally in these tumours, but the expression of INK4a and ARF varied at both the protein and mRNA level, so the contribution of the $C d k n 2 a$ locus to tumour progression in this mouse model needs to be investigated further. Interestingly, Kras $^{\mathrm{G} 12 \mathrm{D}}$ mice that were heterozygous for Tgfbr2 also developed tumours, indicating that $T g f b r 2$ is haploinsuf- ficient. These mice developed more undifferentiated tumours. This might reflect the degree of TGF $\beta$ signalling that remains intact in these tumours, and is similar to the findings in the SMAD4 mouse models in terms of tumour differentiation.

It is intriguing that the loss of Tgfbr 2 or Smad4 can induce different effects on tumour progression and different initial histopathologies. However, the TGF $\beta$ receptors can engage pathways independently of SMAD4. In addition, the loss of SMAD4 also affects signalling from activin and bone-morphogenetic proteins, indicating that these might be involved in the development of PDAC. The continued investigation of these mouse models will hopefully identify more promising targets for the treatment of PDAC.

Nicola McCarthy

ORIGINAL RESEARCH PAPERS Bardeesy, N. et al. Smad4 is dispensible for normal pancreas development yet critical in progression and tumour biology of pancreas cancer. Genes Dev. 15 November 2006 (doi: 10.1101/gad.1478706) | ljichi H. et al. Aggressive pancreatic adenocarcinoma in mice caused by pancreas-specific blockade of transorming growth factor $\beta$-signalling in cooperation with active Kras expression. Genes Dev. 15 November 2006 (doi: 10.1101/gad.1475506)

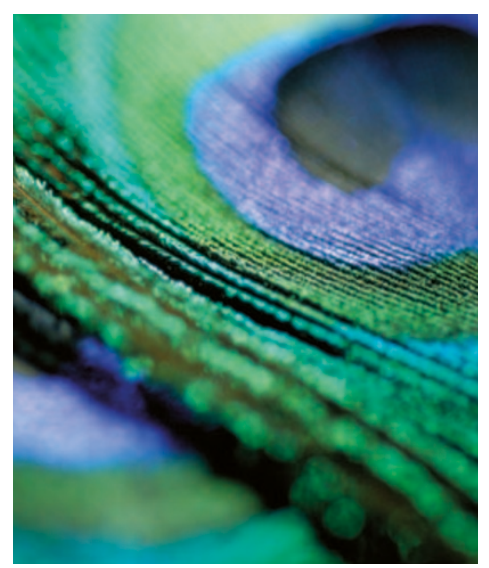

